Portland State University

PDXScholar

\title{
Topological Structures Influencing Kinetic Control in Small, Catalytically Closed, RNA Recombinase Systems Emerging from the Spontaneous Self- Organization of Heterogeneous Fragments of the Azoarcus Ribozyme
}

Sanjay Ramprasad

Portland State University

Follow this and additional works at: https://pdxscholar.library.pdx.edu/honorstheses Let us know how access to this document benefits you.

Recommended Citation

Ramprasad, Sanjay, "Topological Structures Influencing Kinetic Control in Small, Catalytically Closed, RNA Recombinase Systems Emerging from the Spontaneous Self-Organization of Heterogeneous Fragments of the Azoarcus Ribozyme" (2018). University Honors Theses. Paper 607.

https://doi.org/10.15760/honors.618

This Thesis is brought to you for free and open access. It has been accepted for inclusion in University Honors Theses by an authorized administrator of PDXScholar. Please contact us if we can make this document more accessible: pdxscholar@pdx.edu. 


\title{
Topological Structures Influencing Kinetic Control in Small, Catalytically Closed, RNA Recombinase Systems Emerging from the Spontaneous Self-Organization of Heterogeneous Fragments of the Azoarcus Ribozyme
}

\author{
Sanjay Ramprasad \\ Undergraduate Biochemistry Student \\ Department of Chemistry \\ Portland State University \\ Portland, Oregon \\ June 2018 \\ rsanjay@pdx.edu
}

\author{
Principal Investigator and Research Advisor: \\ Dr. Niles Lehman \\ Professor of Chemistry and Biochemistry \\ Department of Chemistry \\ Portland State University \\ Portland, Oregon \\ niles@pdx.edu
}

Thesis document submitted in partial satisfaction of the requirements for the completion of a Bachelor's of Science Honors degree in Biochemistry 


\section{Table of Contents}

Abstract.................................................................3

The Origins of Biological Systems..................................4

Autocatalysis and Self-Replication.................................9

Azoarcus Group I Ribozyme........................................15

Experimental Methods..............................................20

3-Genotype Network Topologies..................................21

Results and Discussion.............................................27

Conclusion................................................................40

References.................................................................42 


\begin{abstract}
It is becoming increasingly evident that at some point, very early in the evolutionary history of terrestrial life, a nascent RNA based chemical system emerged and spontaneously self-organized into hierarchically complex network structures. Recently, it has been mathematically predicted that the architecture of this primitive, prebiotic RNA system (or something very similar) could plausibly provide both the infrastructure and the chemical mechanisms necessary to facilitate a transition to the DNA/protein based biochemical processes universally observed in contemporary biological systems. Complex systems give rise to emergent phenomena through the localized interactions of a large number of agents, at varying scales throughout a network. Moreover, these interactions can be classified topologically, from which it becomes possible to gain insight into the seemingly unpredictable behavior these kinds of systems. Herein, we provide four examples of how the topological artifacts of local interactions between spontaneously self-assembling and self-organizing fragments of the Azoarcus ribozyme can inform both the emergence of decentralized organization and global population dynamics through modulation of kinetic parameters, thereby providing a rudimentary form of selection pressure through which the processes of chemical evolution may occur.
\end{abstract}




\section{The Origins of Biological Systems}

Although a complete chemical explanation for the origins of life on the Earth remains elusive, the last hundred years has seen significant progress towards appreciating the types of chemical processes that may have led to the rise of membrane-bound biological systems capable of metabolism, self-replication, and Darwinian evolution. Recent advances in molecular genomics as well as computer science, computational biology, and bioinformatics have allowed us to delve deeper into the history of life than ever before thought possible. The seminal discovery of the structure of deoxyribonucleic acid (DNA) by Franklin, Wilkins, Watson, and Crick in the 1950s, paved the way for more recent developments molecular biology such as the polymerase chain reaction (PCR), molecular cloning, in vitro evolution, next-generation sequencing, and CRISPR/Cas9. These discoveries have vastly improved our understanding of the transcriptional/translational apparatus.

Nevertheless, increased appreciation of these systems comes part and parcel with new and unique challenges. One particularly vexing new challenge is simply defining the term "life." As additional evidence continues to be uncovered about the necessary requirements of living systems, the distinction between "life" and "non-life" is increasingly obscured. In extant biology, ribonucleic acid (RNA) biosynthesis is almost exclusively dependent on DNA and complex protein machinery in vivo, however recently it is has been demonstrated that an RNA enzyme that acts on oligonucleotide substrates to produce additional copies of itself can be developed through directed in vitro evolution studies [1]. Another investigation, using the autocatalytic Azoarcus group I intron as a 
model system, has shown that certain RNA molecules have the peculiar ability to spontaneously self-assemble from inactive oligonucleotide fragments [2,3]. At the very least, these studies among many others, suggests that RNA possess many of the characteristics retained by higher order biochemical systems - explicitly RNA itself, can comprise a self-sustaining chemical system, with the ability to reproduce and evolve through chemical selection over time.

Modern biochemistry, and as such, contemporary life on Earth requires extraordinarily complex interactions between DNA, RNA, and proteins to sustain itself and reproduce [4]. DNA stores the genetic instructions necessary to produce proteins; similarly the synthesis, repair, and processing of DNA is almost exclusively performed by the very proteins derived from the genetic information stored in DNA. The high degree of interdependence observed in DNA and proteins suggests it is extraordinarily unlikely that either would have ever existed independently of one another for very long (at least in their currently understood chemical forms) [5]. Moreover, given the complexity and specificity of these interactions, it seems even less likely that both species of molecules spontaneously appeared simultaneously on a primitive Earth [5]. It is exceedingly evident when observing the ubiquity of the genetic code and the entire transcriptional/translational apparatus across all domains of life that DNA, RNA, and proteins must have co-evolved from a more primitive genetic/metabolic system(s) that immediately preceded the emergence of life on the Earth. At some point, very early in the evolutionary history of terrestrial life, a self-sustaining chemical system(s) was undergoing a rudimentary form of chemical evolution that likely selected for a transition 
to producing, utilizing, and incorporating DNA and proteins into its pre-existing architecture. This affirms the current scientific consensus that if life arises from inanimate chemicals, it must do so through an intermediary from of pre-biological life that existed closely before. The nature of this pre-biological genetic/metabolic system(s) and how it could have originated is of significant interest to the scientific community at large.

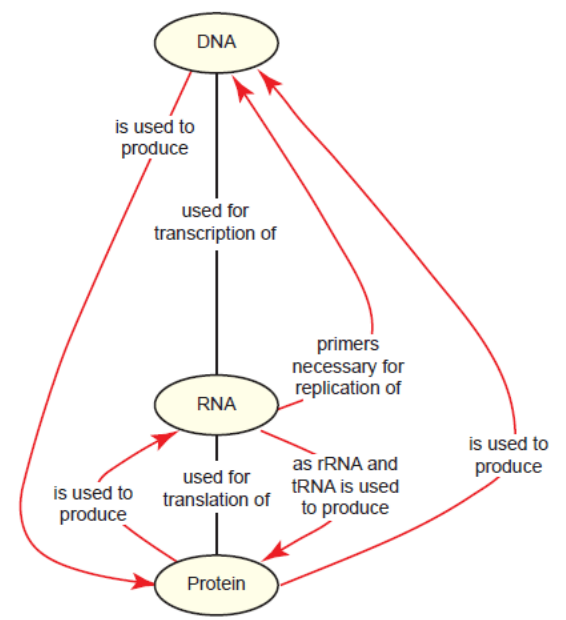

Figure 1: The central dogma of molecular biology depicts information flow in contemporary living systems as largely unidirectional: from DNA through RNA and into proteins. This presents an apparent paradox when conceiving of the origins of the contemporary genetic system, as all three essential macromolecules are simultaneously necessary to be self-sustaining. This is known as a closed, collectively catalytic system.

The question surrounding the origin of the contemporary genetic system is sometimes referred to as the DNA-protein paradox [5]. Figure 1 [5] illustrates this apparent paradox as it relates to the central dogma of molecular biology. In contemporary biochemistry, information flow is largely unidirectional. Protein biosynthesis cannot occur without the information stored in DNA — transmitted through RNA — and DNA synthesis requires the very protein enzymes it codes for. Recently, as our understanding of the role of RNA in 
the genetic system expands, it may seem as if this paradox has become even more confounding. It is now widely appreciated that the central translational organelle in the cell, the ribosome, is indeed a ribozyme (catalytic RNA molecule), and its ability to catalyze peptide bond formation is derived from specialized RNAs known as ribosomal RNAs (rRNAs) [6]. In addition, protein biosynthesis cannot occur without transfer RNAs (tRNAs) that deliver amino acids to the site of protein translation within the cell. It is also well established that RNA processing, an essential feature of RNA biosynthesis, often occurs in an RNA dependent fashion [7,8]. It would appear as though this problem could easily be expanded to be the DNA-RNA-protein paradox. The versatility RNA displays throughout all aspects of contemporary gene expression however, may in fact offer potential insight into how a primitive genetic system(s) may have evolved and functioned.

The notion that RNA or RNA-like molecules may have played a featured role in early prebiotic systems dates back as early as 1967 to a series of papers by Crick, Woese, Orgel and others, in which they hypothesized many of the characteristics of RNA well understood today, including information storage and catalytic functionality [4,9]. Coined the "RNA World Hypothesis," they proposed RNA predated both DNA and proteins in evolutionary history [4,9]. Although catalytic function in RNA molecules was still over a decade away from being uncovered, Orgel and colleagues recognized that the contemporary genetic system was simply too complicated to have arisen de novo from the spontaneous assembly of prebiotic organic molecules [9]. The next logical question was to ask "What could more plausibly become components of a closed, self-replicating system, proteins, RNA, or DNA?" For them, the answer was obviously RNA, as it is the 
only one of the essential biological macromolecules that could conceivably possess both a genotype, and a phenotype. While the debate among scientists continued for many years, the serendipitous discovery of catalytic RNA molecules, or ribozymes, in the early 1980's by Cech and Altman lent increasing credibility to the argument that the current DNA/RNA/protein based biochemical system was almost certainly, immediately preceded by a system(s) in which RNA served as genetic information carriers, metabolic enzymes, and regulatory molecules $[10,11]$. This was further substantiated with the structural determination of the ribosome-showing that rRNAs are indeed responsible for peptide bond catalysis - by Steitz and colleagues in 2000 [6]. As the evidence for an RNA based, nascent genetic/metabolic system(s) continues to grow, questions surrounding the origin and chemistry of an entirely RNA centric, abiotic chemical system remains unresolved. For Orgel, assuming the RNA world was the earliest organized "biochemical" system on a primitive Earth, its origin represents several notable problems as he envisions it:

1. The non-enzymatic synthesis of nucleotides

2. The non-enzymatic polymerization of nucleotides

3. The non-enzymatic replication of RNA molecules

4. The emergence through natural selection of a set of functional RNA catalysts that together could sustain exponential growth

For the purposes of this investigation we focused principally on problem 4 (although the polymerization and replication problems are indirectly addressed); Not all RNA molecules are catalytically functional and the likelihood of the spontaneous polymerization of catalytic RNAs is statistically improbable given the vast sequence space and structural specificity required. What then, if any, are some chemically plausible 
mechanisms for the appearance and evolution of catalytically active RNA molecules in the history of life on the Earth? It seems likely that the answers to questions such as these will greatly advance our collective appreciation for the biochemical processes occurring in present-day biological systems [12]. Thus the question of how small non-functional RNAs originally acquired catalytic activity and eventually evolved into a fully functional abiotic genetic/metabolic system (RNA world) is also of significant interest in contemporary biochemistry and molecular biology. Since very little evidence remains of the primitive proto-life on the Earth, there is tremendous pressure to develop new experimental and theoretical approaches to the study of abiogenesis.

\section{Autocatalysis and Self-Replication}

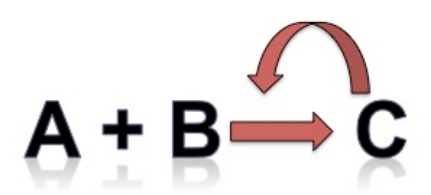

Figure 2: Schematic depicting a hypothetical autocatalytic chemical reaction in which molecule A reacts with molecule $\mathrm{B}$ to produce molecule $\mathrm{C}$, which then can feedback and catalyze its own formation from the reactants $\mathrm{A}$ and $\mathrm{B}$.

While the debate continues surrounding the definition of "life," there are several key features of living systems that have been fairly well established. Chief among them is the notion that life requires the ability to catalyze reactions that lead, directly or indirectly, to the production of more molecules like themselves [12]. From a chemical perspective, the principle mechanism of self-replication is autocatalysis. Autocatalysis, in the simplest sense, is when the product of a chemical reaction can catalyze its own formation from the original reactants while remaining chemically stable in the process (Figure 2). 
Autocatalytic reactions are by no means exclusive to biology and are often observed in many abiotic chemical systems. A specific example is the redox reaction of permanganate with oxalic acid. This reaction is catalyzed by $\mathrm{Mn}^{2+}$ ions and when potassium permanganate reacts with oxalate in low $\mathrm{pH}$ conditions, a product is the $\mathrm{Mn}^{2+}$ ion as shown in the balanced chemical equation below:

$2 \mathrm{KMnO}_{4}^{-}(\mathrm{aq})+5 \mathrm{C}_{2} \mathrm{H}_{2} \mathrm{O}_{4}(\mathrm{aq})+6 \mathrm{H}_{3} \mathrm{O}^{+} \rightarrow 2 \mathrm{Mn}^{2+}(\mathrm{aq})+2 \mathrm{~K}^{+}(\mathrm{aq})+10 \mathrm{CO}_{2}(\mathrm{~g})+14 \mathrm{H}_{2} \mathrm{O}(\mathrm{I})$

Initially, the rate of this reaction is quite slow at room temperature, however as the concentration of $\mathrm{Mn}^{2+}$ ion increases in solution as the reaction proceeds, the rate at which oxalate is oxidized to carbon dioxide also increases. It is important to note here that all catalysts, whether they are inorganic, organic, or biological, act merely to lower the activation energy required for the reaction to take place. Catalysts cannot influence thermodynamic parameters and the free energy associated with both the reactants and the products remains fixed regardless of whether the reaction was catalyzed or not. In other words, catalysts can only accelerate the rate of a spontaneous reaction and will have no effect on thermodynamically disfavored processes.

A collection of autocatalytic reactions can be considered a collectively autocatalytic system (CAS) if the molecules and the chemical reactions between them form a closed, self-sustaining network $[13,14]$. The notion that autocatalytic sets could plausibly provide the organization necessary for the emergence of life is not novel, and was proposed as early as 1978 by Eigen and Schuster [15]. In the 40 years hence, significant progress has been made towards formally understanding the fundamental chemical dynamics of 
collectively autocatalytic systems both through mathematical models, and in engineered laboratory systems $[2,3,9,1314,15,16,17,19,20,21,22]$. An autocatalytic set, or collectively autocatalytic system (CAS), can be formally described mathematically [13]:

$$
\text { Define }(\mathrm{CAS}) \mathrm{Q}=\{\mathrm{X}, R, \mathrm{C}\}
$$

$$
\begin{array}{ll}
\text { - } & \mathrm{F}=\left\{\mathrm{f}_{1}, \mathrm{f}_{2}\right\} \\
\text { - } & \mathrm{X}=\mathrm{F} \cup\{\mathrm{M} j \mathrm{~N} \mid \mathrm{M}, \mathrm{N} \in\{\mathrm{A}, \mathrm{C}, \mathrm{G}, \mathrm{U}\} \\
\text { - } & R=\{\mathrm{M} j \mathrm{~N} \mid \mathrm{M}, \mathrm{N} \in\{\mathrm{A}, \mathrm{C}, \mathrm{G}, \mathrm{U}\} \\
\text { - } & \mathrm{C}=\left\{\left(\mathrm{M}_{1} j \mathrm{~N}_{1}, \mathrm{M}_{2 j} \mathrm{~N}_{2}\right) \mid \mathrm{M}_{i} \mathrm{~N}_{i} \in\{\mathrm{A}, \mathrm{C}, \mathrm{G}, \mathrm{U}\}, i \in\{1,2\} \&\right. \\
& \left(\mathrm{M}_{1} \mathrm{~N}_{2}\right) \in\{(\mathrm{A}, \mathrm{U}),(\mathrm{U}, \mathrm{A}),(\mathrm{G}, \mathrm{C}),(\mathrm{C}, \mathrm{G})\}
\end{array}
$$

where some CAS, Q, is composed of the elements X (defined as the set of environmental cofactors such as divalent metal ions (F) and substrate RNA molecules), $R$ (the collection of reactions sustaining the system), and $\mathrm{C}$ (the set of ribozymes that facilitate the reactions in the system) [13]. This mathematical structure has some nice properties that make it a fairly good model for abstracting a generalized RNA based self-replicating system. First, it is displays catalytic closure, that is to say that no reaction takes place within the system that is not catalyzed by a subset of $\mathrm{C}$ (say $\mathrm{C}^{\prime}, \mathrm{C}^{\prime \prime}$, etc.). Additionally, the structure is self-sustaining, as all of the necessary reactants, catalysts, products—and the reactions that produce them-are contained within Q. Although this structure represents a respectable model for catalytic RNA systems, mathematical abstractions are not necessarily governed by the natural physical/chemical constraints imposed on real world systems, thus the scope of an abstraction can quickly become so immense, computational analysis represents a significant challenge. Moreover, any specific constraints imposed on the system must be strictly defined. This is often difficult, given the current understanding of molecular phenomena is thus far incomplete. Nonetheless, 
mathematical models such as these do provide a preliminary starting point from which specific properties may be observed empirically.

The strength of this, and other mathematical models, lies in the ability to analyze a large number of distinct CAS through computational methods [13]. Computation can serve to identify specific properties that may apply broadly to all similar systems. This is something that is far more challenging in laboratory systems given the time and resource requirements to exhaustively test enough examples to draw statistically significant inferences. An algorithm [13] has been created to explore the above mathematical structure and along with recent experimental data $[2,3,14,20]$, a richer appreciation for how catalytically closed systems arise, propagate, and evolve is beginning to emerge.

Autocatalytic sets and systems of self-replicators have demonstrated, both in computer simulations and in constructed laboratory systems, to display some remarkably "life-like" properties:

1. Autocatalytic sets spontaneously emerge given there is adequate complexity within a chemical system. While there is still some debate concerning this particular feature, recent studies have shown, both theoretically and computationally, a linear growth rate in the level of catalysis in a chemical system is sufficient for the spontaneous formation of autocatalytic sets [16]. Furthermore, the validity of these computational models has been, in part, corroborated by work from Vaidya et al. demonstrating that autocatalytic cycles spontaneously arise in vitro from 
non-functional oligonucleotide fragments of a self-assembling, selfreplicating ribozyme based on the Azoarcus group I intron [17]. These studies suggest that self-organization - in the absence of higher order control - might even be unavoidable in sufficiently complex chemical systems.

\section{Self-replicating systems possess the potential to undergo evolution}

through selection pressure. Although all physical systems are globally governed by the second law of thermodynamics - which states that all closed physical processes tend towards the state of lowest free energy and maximal entropy, i.e. equilibrium—systems of self-replicators are driven towards dynamic kinetic stability instead [18]. The qualitative distinction between thermodynamic stability and kinetic stability is that closed systems, at thermodynamic equilibrium, will remain unchanged over time (static stability); kinetically driven systems however, achieve a dynamic stability when the rate of product formation equals the rate of product decay. This gives kinetically stable systems the freedom to change (if possible) so long as the rate of product formation and product decay remains equal. Dynamic kinetic stability is observed in self-replicating systems as a consequence of their ability to grow exponentially [18]. If the product of every reaction is itself a catalyst for the same reaction, one can imagine it will not be too long before the number of self-replicators (catalysts) far outnumbers the available substrates (resources), and accordingly results in substantially increased competition for reproduction 
within the closed system. To draw an analogy to biology, the ability to achieve kinetic stability in self-replicating systems can be considered a fitness parameter, or selection pressure driving the chemical evolution of these types of systems [18].

\section{Hierarchical organization is an emergent property of autocatalytic sets.} Autocatalytic sets often display complex, recursive, modular, organizational structure, in which the entire system itself, can be composed of many closed autocatalytic subsets [19]. If autocatalytic sets can indeed be decomposed further into autocatalytic subsets, which in turn are also composed of autocatalytic sub-subsets and so on, it would appear as if higher level, functionally closed structures are an emergent characteristic of these systems [19]. This property has also been demonstrated in empirical studies using the Azoarcus group I ribozyme [17]. Furthermore, these higher levels of organization suggest it is possible to reproduce autocatalytic systems through partitioning. If an autocatalytic set were segregated into two discrete systems, in selected cases, it is mathematically predicted the same system of reaction networks could spontaneously reform in time, creating a second copy of the system (although the two resulting systems would be half the size as the original) [19]. This type of global reproduction can even be imagined as a rudimentary form cellular mitosis predating the evolution of membranebound chemical systems [24]. A hierarchical organizational structure has important consequences for the growth, persistence, and evolvability of 
any sufficiently complex system, and therefore may represent an absolute requirement for the evolution of life $[19,24]$. Take for example, the assembly of a car that is composed of tens of thousands of individual parts. If a single person is solely responsible for the assembly of these vast arrays of parts, it is highly unlikely a functional car would ever be produced. Some parts would get lost and yet others would not remain stable without other supporting structures securing them in place. However, if the car was first assembled into small modules along an assembly line, which in turn can be used to assemble larger modules and so on, eventually a functional car would emerge — through modular design and hierarchical organization. Another example from abstract mathematics is the Mandelbrot Set. Fractals such as these demonstrate the vast complexity that can arise from relatively simple recursive algorithms. The great power of recursivity lies in the ability to define an infinite set of objects from a finite set of instructions [25].

\section{The Azoarcus Group I Ribozyme}

For the current investigation, the covalently self-assembling Azoarcus pre-tRNA ${ }^{\text {Ile }}$ group I intron is used as a model prebiotic system. Many eukaryotic-and some archaeal and bacterial-RNAs contain long, non-coding intervening sequences (introns) after transcription. During RNA biosynthesis, these introns must be removed concomitant with the recombination of the coding sequences (exons). The majority of introns are excised from pre-RNA transcripts by large RNA/protein splicing complexes to produce mature 
RNA, however group I introns are autocatalytic ribozymes, that can self-excise from RNA primary transcripts. In vivo, these ribozymes fold, through base pairing and stacking interactions, into catalytically active, three-dimensional structures that can facilitate their own excision and recombination of the $5^{\prime}$ and $3^{\prime}$ exons. The threedimensional structure of the ribozyme orients the scissile phosphodiester bonds at the splice site (SS) into the active site along with two $\mathrm{Mg}^{2+}$ ions. A fundamental feature of the folding event is the formation of the internal guide sequence (IGS). The IGS recognizes the splice site on the $5^{\prime}$ exon and orients it into the active site with an exogenous guanosine cofactor for the first step of the reaction, then aligns the newly liberated $3^{\prime} \mathrm{OH}$ on the $5^{\prime}$ exon with the $3^{\prime}$ splice site for the second step. Consensus sequences in three locations, the $5^{\prime} \mathrm{SS}, 3^{\prime} \mathrm{SS}$, and the IGS specify the exact locations of the splicing reactions.

The self-splicing reactions of group I introns occur in two sequential, trans-esterification reactions in which the intron is spliced out of the nascent RNA transcript concurrent with the covalent recombination of the two flanking exons. The intrinsic chemical competency of group I introns to form and break phosphodiester bonds can be harnessed in vitro to develop a putative self-replicating system $[17,20]$. The ability to catalyze recombination reactions such as these, make group I introns likely candidates to model prebiotic systems, since the origin of life necessitates a chemical mechanism—preferably incurring minimal to no thermodynamic cost—-for information containing chemicals to form heterogeneous populations [20]. 
The Azoarcus pre-tRNA ${ }^{\text {Ile }}$ group I intron (Figure 3a) is 203 nucleotides (nt) in length, and can spontaneously re-assemble from two, three, or four fragments of itself when incubated at $48^{\circ} \mathrm{C}$ in a $\mathrm{MgCl}_{2}$ solution [2,3]. The self-assembly reaction occurs via a transient complex, associating through hydrogen-bonding interactions, that retains enough residual catalytic activity to assemble a covalently contiguous ribozyme $[2,3]$. The full-length ribozyme is then capable of catalyzing the recombination of two exogenous RNA fragments to replicate quite robustly [2,3]. The recombination reaction is mediated through a 3-nt base pairing interaction, between the IGS and its 3-nt compliment sequence on a substrate oligonucleotide, which can be mutated to alter the specificity and rate of the recombination reactions that occur within a system [20]. Through specific single nucleotide substitutions, in the middle position of both the IGS and its compliment, it is possible to direct the catalytic activity of mutant Azoarcus ribozyme fragments through spontaneous network evolution [17]. This investigation utilizes a two-piece assembly system where the full-length Azoarcus ribozyme is split into two fragments: a 148-nt fragment symbolized as 5'-WXY-3', and a 55-nt fragment represented as $\mathbf{Z}$ (Figure 3a). The complete self-assembly reaction can be represented as:

\section{$\mathrm{WXY}+\mathrm{Z} \rightarrow \mathrm{WXYZ}$}

The WXY fragments are mutated by altering one of the first $\left(5^{\prime}\right)$ three nucleotides in the $\mathbf{W}$ region corresponding to its IGS, and one of last (3') three nucleotides in the $\mathbf{Y}$ region, corresponding to its compliment, which is recognized by the IGS of the catalytic ribozyme assembling the two fragment oligonucleotides. The mutant genotypes are characterized by these two nucleotide substitutions (denoted as $\mathrm{M}$ and $\mathrm{N}$ in Figure 3a). In the wildtype ribozyme, the IGS is $5^{\prime}-\mathrm{GUG}-3^{\prime}$ and its compliment is $5^{\prime}-\mathrm{CAU}-3^{\prime}$ [23]. A 
mutant engineered with an IGS of $5^{\prime}$-GGG-3' and a compliment of 5'-CCU-3' would be referred to as genotype $\mathbf{G C}[2,3,17,21]$.

Since these are self-replicating ribozymes, it becomes possible to pit different genotypes of WXY fragments against each other to compete for a common resource $(\mathbf{Z})$ for reproduction. When forced to compete to self-replicate in a reaction network, the fragments "auto-select" an optimal reproductive strategy categorized as "selfish" or "cooperative," based on the kinetic dynamics of the global network (Figure 3b) [21]. A cooperative strategy is defined as one where a specific genotype is kinetically driven to assemble another ribozyme of a distinct genotype, resulting in heterogeneity within the population [21]. Conversely, a selfish strategy is simply the further propagation of a single genotype, resulting in homogenous populations [21]. Thus the population dynamics of this chemical system is manifest by the interplay of selfish and cooperative interactions [21]. This type of kinetic competition among molecules capable of storing and transmitting genetic information was likely an essential feature of sufficiently complex chemical systems before the advent of the Darwinian type of evolution observed in contemporary biological systems [21,22]. Moreover, this suggests that diverse interactions on the molecular level (through kinetic parameters) strongly influence the evolution of global population dynamics [17]. A greater appreciation of the types of selfish and cooperative interactions possible in small self-replicating networks may lend insight into how the larger heterogeneous RNA populations, required for a fully functional RNA world, might have evolved during the nascent stages of terrestrial life [21]. 
a

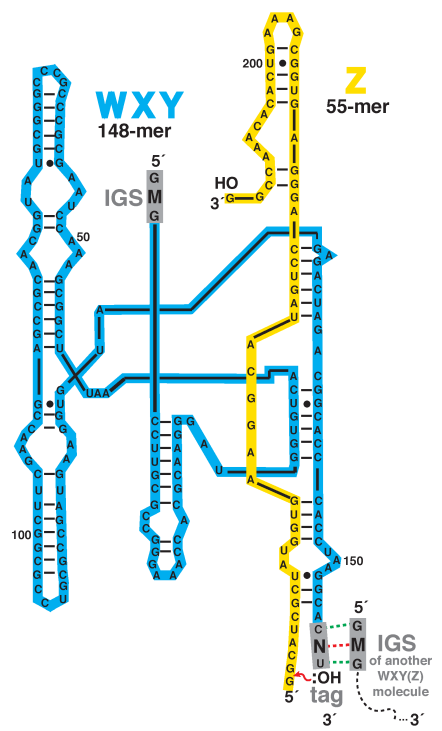

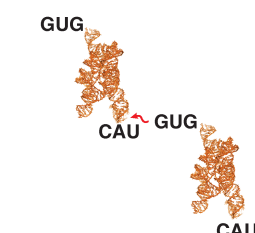

selfish

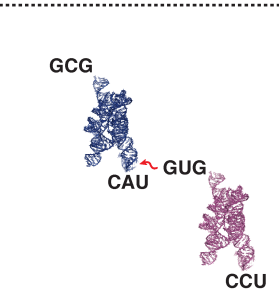

cooperative

Figure 3: The Azoarcus catalytic RNA, covalently self-assembling system. (a) A schematic of the reaction between the previously described WXY and $\mathbf{Z}$ fragments depicting the interaction between the IGS of the ribozyme catalyzing the assembly, and its compliment triplet on the 3' end of the WXY fragment. (b) Two examples demonstrating the difference between selfish assembly strategies $v i s-\grave{a}$-vis cooperative assembly strategies [21].

In this manuscript, we begin by graphically classifying the pairwise interactions of all possible, 3-edge, 3-node, catalytic network systems that could emerge from selforganizing fragments of the Azoarcus ribozyme, into 17 topologically distinct, architectural families. Moreover, we have characterized four of these predicted catalytic network architectures, and evaluated their viability as foundational cores of an evolvable CAS, through empirical study of the Azoarcus Recombinase System. Furthermore, we will attempt to show that these topological artifacts are kinetically determined through localized connections that can be rationalized on the molecular level through known mechanisms of RNA:RNA mediated interaction. Finally, we comment on how network architecture may influence global population dynamics within the characterized triplet systems. 


\section{Experimental Methods}

RNA sample preparation: The WXY molecules, which are 148-nt fragments of the Azoarcus group I intron, were prepared using standard in vitro transcription protocols. Single stranded DNA plasmid templates, purchased through TriLink BioTechnologies, were assembled via Thermococcus litoralis (Vent) DNA polymerase assisted template reconstruction and then amplified through polymerase chain reaction (PCR). Site-specific mutations arise from distinct primers containing the desired nucleotide substitution, introduced during the PCR protocol. The assembled double stranded DNA PCR products were then ethanol precipitated and rehydrated in RNase free, reaction grade $\mathrm{H}_{2} \mathrm{O}$, to desired volume. Run-off transcription was performed using T7 RNA polymerase, in transcription buffer along with equal concentrations of all four ribonucleotide triphosphate molecules (ATP, GTP, UTP, CTP) plus rehydrated PCR products, and incubated at $48^{\circ} \mathrm{C}$ for approximately $6-8$ hours. After incubation, the transcribed RNA is separated via dual phase phenol/chloroform-IAA organic extraction, ethanol precipitated, then purified through polyacrylamide gel electrophoresis (PAGE). Purified RNA molecules were resuspended in $0.1 \mathrm{mM}$ EDTA and diluted to $10 \mu \mathrm{M}$ concentrations. $\mathbf{Z}$ molecules were purchased directly from TriLink BioTechnologies.

A small fraction of the WXY molecules were radiolabeled for visualization and quantification via $5^{\prime}$-labeled $\gamma\left[{ }^{32} \mathrm{P}\right] \cdot$ ATP added post-transcription using OptiKinase.

Serial Transfer Network Experiments: A master mix was prepared containing $\mathbf{W} \mathbf{X} \mathbf{Y}_{\mathrm{A}}$ $(1.0 \mu \mathrm{M}), \mathbf{W X Y} \mathbf{Y}_{\mathrm{B}}(1.0 \mu \mathrm{M})$, and $\mathbf{W X Y} \mathbf{Y}_{\mathrm{C}}(1.0 \mu \mathrm{M})$, and $\mathbf{Z}(3.0 \mu \mathrm{M})$. The master mix was then divided into three equal volumes, and doped with ${ }^{32} \mathrm{P}$-labeled WXY $(<0.1 \mu \mathrm{M})$. One tube was doped with labeled $\mathbf{W X Y} \mathbf{Y}_{\mathrm{A}}$, the second with labeled $\mathbf{W} \mathbf{X} \mathbf{Y}_{\mathrm{B}}$, while the third was 
doped with labeled $\mathbf{W X Y} \mathbf{Y}_{\mathrm{C}}$. Each tube containing labeled $\mathbf{W X Y}$ molecules were then aliquoted into eight, 200- $\mu$ l PCR tubes. Each reaction set was therefore composed of 24 small PCR tubes, eight for each genotype being observed in the system. The reaction tubes were then heated to $80^{\circ} \mathrm{C}$ for 2 minutes, and subsequently cooled to $48^{\circ} \mathrm{C}$ for the duration of the experiment. The reaction in tube 1 (of 8) was initiated with reaction buffer (100 mM MgCl $2,30 \mathrm{mM}$ EPPS, $\mathrm{pH} 7.5$ ) and allowed to proceed for 5 minutes, at which point $10 \%$ of the solution volume from tube 1 was transferred to tube 2 and initiated with reaction buffer concomitant with the quenching of reaction tube 1 on ice and with the addition of an equal volume of quench solution (1:3 ratio of 0.5 M EDTA and 2X PAGE loading dye with urea). This transfer protocol was repeated every 5 minutes through eight transfers. A small portion of the original three reaction mixtures containing the labeled WXY were kept as a $t=0$ time points. The samples were then loaded on an $8 \%$ polyacrylamide/8M urea gel to separate $\mathbf{W X Y}$ from $\mathbf{W X Y Z}$ molecules. Visualization and quantification was obtained through phosphorimaging on a Typhoon Trio+ variable mode

phosphorimager along with ImageQuant software. The extent of the reaction in each tube was calculated as percent (\%) reacted by the following equation:

$$
\% \text { reacted }=[\text { reacted value } /(\text { reacted value }+ \text { unreacted value })] * 100
$$

The acquired data was then plotted as a function of transfer number for each of the three genotypes monitored over the duration of the experiment.

\section{3-Genotype Network Topologies}

Traditional scientific approaches (at least in the physical sciences) to understanding macroscopic phenomena, seek to uncover how isolated properties intrinsic to the 
foundational units (subatomic particles, atoms, molecules, cells, etc.) of a larger system can, in totality, produce the collective behavior in question [21]. This type methodological reductionism has, without a doubt, historically served the processes of scientific inquiry quite well. In some contexts however, these types of approaches have been less successful. A particularly notable challenge has been in rationalizing and predicting the emergence of complex systems.

What distinguishes emergent complex systems (biology), from merely significantly complicated systems (airplane), is that complex systems tend to exhibit global behavior that is seemingly impossible to predict from the observation or modeling of behavior on an elemental scale. This property is a consequence of non-linear, spatial, and time dependent interactions, among large numbers of local agents and subsystems, within an emergent complex system [26]. The difficulty in modeling and predicting non-linear dynamics arises from the effect small perturbations have on the system as a whole. In linear systems, small disturbances to local regimes correspond to proportionally small disturbances to the global regime. Non-linear systems, by contrast, are highly sensitive to initial conditions, and react unpredictably to small perturbations. This is because tiny, dynamical fluctuations are often exponentially amplified through the recursive organizational structure, or motifs (positive feedback cycles, feed forward loops, etc.), that are characteristic of these types of systems. While a precise definition of emergent complexity continues to be disputed throughout many disciplines, virtually every working definition discussed in the literature implies the existence of the sorts of hierarchical, self-organizational structures, required to produce these motifs [27]. 
The decentralized organization of complex systems develops from the local interdependence of many elements within the network. These localized patterns of interdependence then begin to interact with other locally interdependent networks, giving rise to whole new levels of organization. Arrangements of nested orders of organization are seemingly omnipresent characteristics of natural, technological, and social systems [28]. A ubiquitous feature of many of these systems is highly dense clusters of connectivity $[29,30]$. Just as ordered levels of localized interdependence can give rise to hierarchical organizational structure, the interdependence between global, regional, local, and sub-local networks (nodes), emerges from the density of the connections that span them [30]. The clustering of pairwise interactions between elements therefore modulates the degree of interdependence - which in turn determines the degree of structural organization observed within the system. As the components of a system become increasingly connected, it becomes the nature and topology of these connections that define the system, rather than the fundamental properties of the constituent elements $[29,30]$.

The structural architecture of a network system —ultimately a consequence of topological properties at various levels of interaction - can be well described by mathematical graphs [27]. The branch of discrete mathematics concerned with the structures and properties of graphs is known as graph theory. Informally, a graph is simply a discrete collection of elements (nodes), which exhibit a collective interdependence defined by pairwise interactions that can be represented by arrows (edges) connecting the space between 
them. More formally, a graph is a mathematical structure that satisfies the following criteria:

$$
\text { Define (Graph) } \mathbf{G}=\{\mathrm{V}, \mathrm{E}, f\}
$$

where a graph $(G)$ is an ordered triple that contains of a set of vertices $V(G)$, a set of edges $\mathrm{E}(\mathrm{G})$, and a set of transformations $f$, such that $\mathrm{V}(\mathrm{G})$ and $\mathrm{E}(\mathrm{G})$ are both non-empty and finite and $f$ maps each element in $\mathrm{E}(\mathrm{G})$ to an unordered pair of vertices in $\mathrm{V}(\mathrm{G})$ [31]. In a graph, two vertices $\mathrm{V}\left(\mathrm{G}^{\prime}\right)$ and $\mathrm{V}\left(\mathrm{G}^{\prime \prime}\right)$ are known as adjacent if and only if, there is an edge $\mathrm{E}\left(\mathrm{G}^{\prime}\right)$ that connects them. Then a vertex $\mathrm{V}\left(\mathrm{G}^{\prime}\right)$ is said to be incident to the edge $\mathrm{E}\left(\mathrm{G}^{\prime}\right)$ if and only if, $\mathrm{V}\left(\mathrm{G}^{\prime}\right)$ is adjacent to $\mathrm{V}\left(\mathrm{G}^{\prime \prime}\right)$. The degree of a vertex $\mathrm{V}\left(\mathrm{G}^{\prime}\right)$ is the number of edges in $E(G)$ incident with $V\left(G^{\prime}\right)$. The degree of a vertex is a quantitative measure of the connectivity of a particular vertex in a graph. Finally, two graphs, $\mathbf{G}_{\boldsymbol{i}}$ and $\mathbf{G}_{i i}$, are said to be topologically identical or isomorphic if and only if, there is a one-toone correspondence between $\operatorname{deg} \mathrm{V}\left(\mathbf{G}_{\boldsymbol{i}}\right)$ and $\operatorname{deg} \mathrm{V}\left(\mathbf{G}_{i i}\right)$.

Graph theoretic principals, which incorporate elements from the related fields of topology, combinatorics, and algebra, can be tremendously powerful tools for exploring how higher order organizational structures arise from local interactions. During this investigation we were keen on utilizing a graph theoretic approach to modeling the small, 3-genotype network systems that arise from the spontaneously, self-organizing oligonucleotide fragments of the Azoarcus ribozyme. For this system, a simple 3genotype network can be represented as a graph, where each vertex (or node) represents a distinct genotype in the system, and the edges represent the direction of catalytic activity (reproductive strategy) of those genotypes. The transformation of a 3-genotype system 
into a network graph produces a topological representation of the kinetic conditions that emerge and drive the global population dynamics of each genotypic triplet set. Within each triplet set, a single genotype node can employ either or both of two reproductive strategies depending on the kinetic constraints of the system:

1. A selfish strategy, in which one genotype catalyzes the assembly of ribozymes of the same genotype-represented graphically by a self-loop. 2. A cooperative strategy, in which one genotype catalyzes the assembly of ribozymes of a distinct genotype - represented by an edge that spans from the node of the genotype responsible for assembly to the node of the genotype being assembled.

To determine which reproductive strategies a given node employs, a three by three matrix was constructed for each triplet system using previously determined experimental autocatalytic rate constants [20]. These rate constants were then used to extrapolate the rate constants of cross-assembly. This way we were able to compare the kinetic efficiency of all of the possible reproductive strategies - from empirical data - between three genotypes, for any given triplet set. The three strategies from each set with the greatest kinetic efficiency ultimately defined the edge topologies (internode or intranode) of the system.

There are 16 possible Azoarcus WXY genotypes that can be engineered by altering the identity of the $M N$ residues (Figure 3a), corresponding the middle nucleotide residue of the IGS (5' region of $\mathbf{W X Y}$ ) and the middle nucleotide residue of its compliment sequence $\left(3^{\prime} \mathbf{W X Y}\right)$, to any of one of the four canonical ribonucleotides $(A, C, G, U)$ 
$[20,21]$. Previous studies have demonstrated the evolvability of three-genotype networks based on the Azoarcus recombinase system, by characterizing a system that represents a "rock-paper-scissors" (RPS) topology (Figure 4.2) [20,21]. RPS, however, is only 1 of 17 possible, non-trivial, topologically distinct, architectural families that can emerge from a three-node, three-edge system (Figure 4) [21]. From the 16 Azoarcus genotypes, twentysix, 3-genotype triplet sets were examined and were successfully categorized into 10 of the 17 possible network structure classes best describing their kinetically determined, organizational structure. Given that for 16 possible genotypes of the Azoarcus system there are 560 possible, 3-genotype permutations, and given that investigation into only twenty six of them were illustrative of 10 out of the 17 structural classes, it seems highly likely further evaluation of the remaining 534 triplet sets would provide representative examples of the remaining 7 classes of network topologies.

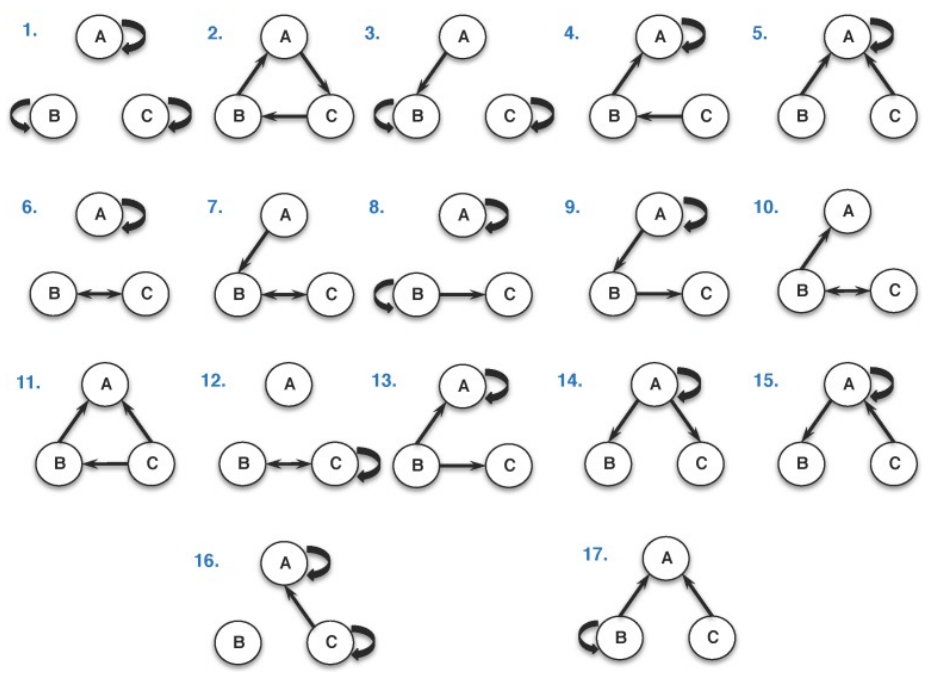

Figure 4: The 17 possible, non-trivial, topologically distinct, 3-edge, 3-node networks structure classes. In these diagrams, A, B, and $\mathbf{C}$ represent one of the 16 Azoarcus WXY genotypes, while the arrows of each individual graph represents the three most kinetically efficient reproductive strategies within the system. Arrows that begin and terminate at the same node (self-loop) denote a selfish strategy, whereas arrows that begin at one node and terminate at a distinct node denote a cooperative strategy [21]. 


\section{Results and Discussion}

Genotype Triplet Catalytic System: UC, GU, \& CA

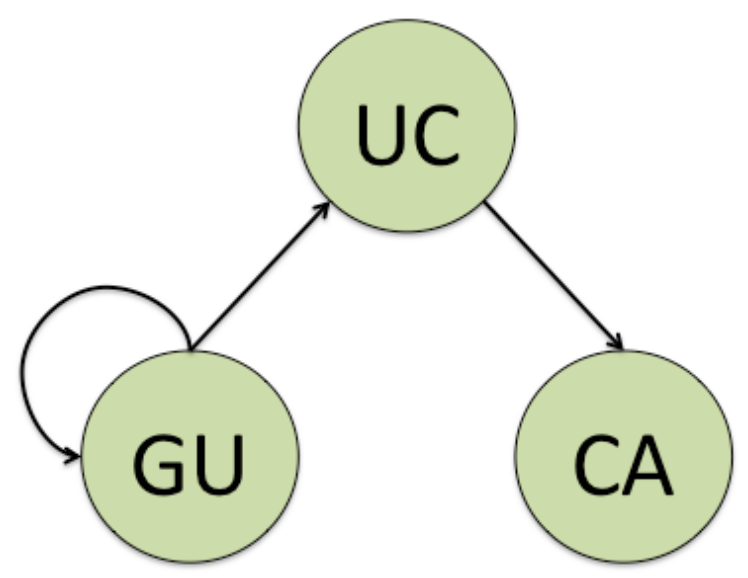

Figure 5: The predicted topological network structure of the genotype triplet UC/GU/CA catalytic system as determined by experimentally determined catalytic rate constants [20] of assembly. This system is topologically organized through an autocatalytic (selfish) node nested within, and upstream of, a 3-node, cooperative cascade.

Figure 5 illustrates the predicted topological architecture of the genotype triplet $\mathrm{UC} / \mathrm{GU} / \mathrm{CA}$ catalytic system. This network is organized through three $M N$ nucleotide, base pairing interactions: a $\mathrm{G} \cdot \mathrm{U}$ wobble base pair, mediating the self-assembly of genotype $\mathbf{G U}$, a $\mathrm{G} \bullet \mathrm{C}$ Watson-Crick base pair mediating the cross-assembly of genotype UC by genotype $\mathbf{G U}$, and a $\mathrm{U} \bullet A$ Watson-Crick base pair, mediating the cross-assembly of genotype CA by genotype UC. The rate constant for the self-assembly of genotype GU is $0.0091 \mathrm{~min}^{-1}$, the rate constant for the cross-assembly of genotype $\mathbf{U C}$ is 0.0125 $\min ^{-1}$, and the rate constant for the cross-assembly of genotype CA is $0.0197 \mathrm{~min}^{-1}$. Although the rate of self-assembly for genotype $\mathbf{G U}$ is an order of magnitude smaller than those of both the cross-assembly connections depicted in the above structure, the relative kinetic advantage of $\mathbf{G U}$ self-assembly vis-à-vis the other 6 possible catalytic 
pathways available to this network strongly suggests this triplet set can organize into a collectively autocatalytic system (CAS). A CAS is a catalytically closed, self-sustaining, network that can collectively reproduce the constituent elements [13]. The topological architecture of the above triplet set predicts these conditions can be satisfied in this system, at least for some finite period of time. Moreover, this network system demonstrates some putative viability as a CAS foundational core as it features fairly moderate connective density through one node of degree two, and two nodes of degree one, giving the entire system an overall connectivity degree of 4 , only 1 degree higher than the minimum. It is difficult to speculate however, about how robust this system truly is, as the topology seems to indicate that a perturbation of the $\mathbf{G U}$ node, particularly early in the reaction scheme, may disrupt the cooperative cascade of assembly pathways rendering the CAS non-viable.

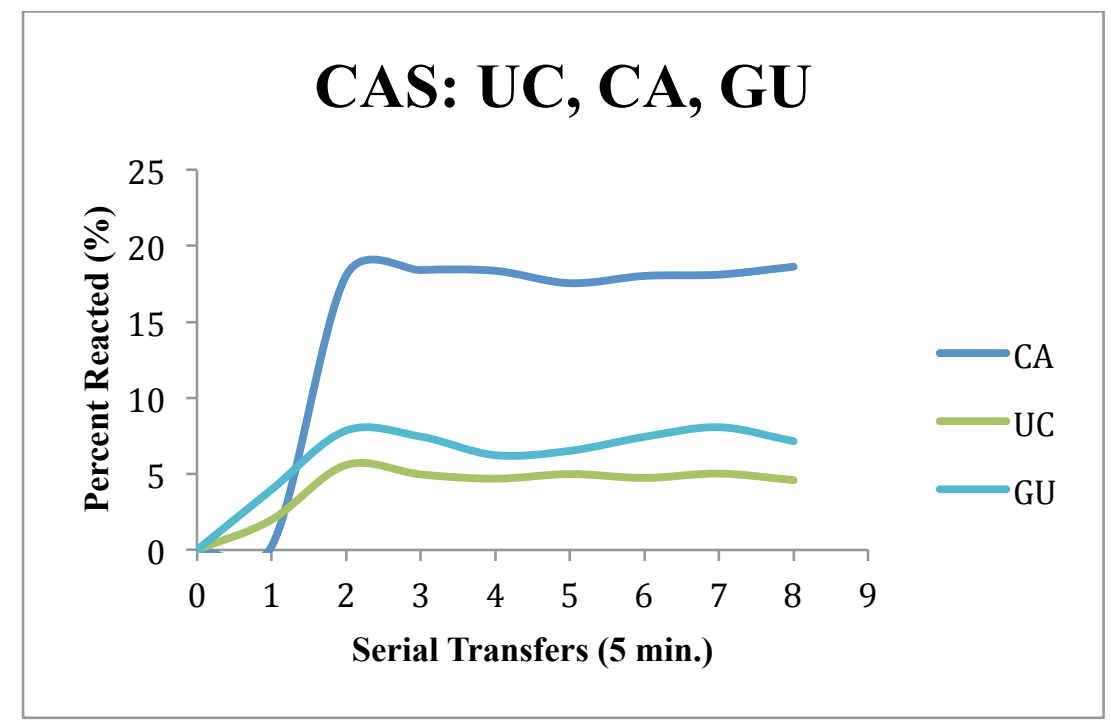

Figure 6: Empirical result from the serial dilution experiment. Catalytic network system composed of the WXY genotypes UC/CA/GU. This plot demonstrates the evolving population dynamics of this triplet set over the course of 8 serial transfers and fluctuations in population diversity are expressed as a percent of original WXY molecules that are assembled into catalytically active WXYZ ribozymes. 
A plot of the global population outcomes from the serial dilution experiment of the $\mathrm{UC} / \mathrm{CA} / \mathrm{GU}$ triplet set as a function of 8 serial transfers over the duration of 40 minutes (Figure 6). Changes in the global population distribution are represented as the variation in the overall percent of the original WXY fragments that get assembled into full length, WXYZ ribozymes. This result demonstrates a clear divergence in the genotypic population distribution, within this set, over the first and second transfers, occurring between 5 and 10 minutes of reaction time. This is, in fact not terribly surprising, as the topological architecture suggests, assembly reactions downstream of the $\mathbf{G U}$ node are not kinetically favored until the concentration of WXYZ-GU reaches a critical threshold level, observed around 5\% reacted, at which point the cascading cooperative assembly reactions become more kinetically facile and dominate the system. However, before the first transfer, and within the initial 5 minutes of the reaction, GU self-assembly is the dominant catalytic pathway in this system concurrent with $\mathbf{U C}$ cross-assembly by $\mathbf{G U}$, however to a noticeably lesser extent. These findings could suggest that early on, when the populations of all three $\mathbf{W X Y Z}$ genotypes are close to equal, and systemic competition for resources is minimal; the selfish, autocatalytic node has an apparent kinetic advantage. As soon as the environmental conditions change however, and the concentration of WXYZ-GU surpasses that of the other two genotypes, the cascading cooperative pathway becomes kinetically preferred. A plausible rationalization for the apparent kinetic advantage of WXYZ-GU early on, is that during saturating conditions, i.e. when substrate concentrations exceed ribozyme concentrations, the reduced thermodynamic stability of a $\mathrm{G} \bullet \mathrm{U}$ wobble pair has less of an effect on the ability of the 
ribozyme to successfully bind substrate WXY molecules, given that potential substrates are relatively plentiful. However when an asymmetry arises in the statistical distribution of substrates throughout the system, resulting from a local decrease in the concentration of WXY-GU molecules (or the relative local increase in the concentration of WXY-CA), the more thermodynamically stable $\mathrm{G} \cdot \mathrm{C}$ Watson-Crick interaction provides greater substrate affinity, thus favoring the GU-CA cross assembly pathway, which in turn accelerates the downstream cascade. Finally, it is not surprising that once this cascade pathway is initiated, WXYZ-CA is the ultimate beneficiary, as it has the greatest inherent kinetic advantage from an experimental rate constant that is greater than either of the other two catalytic assembly pathways.

Genotype Triplet Catalytic System: $C A, A C, \& G U$

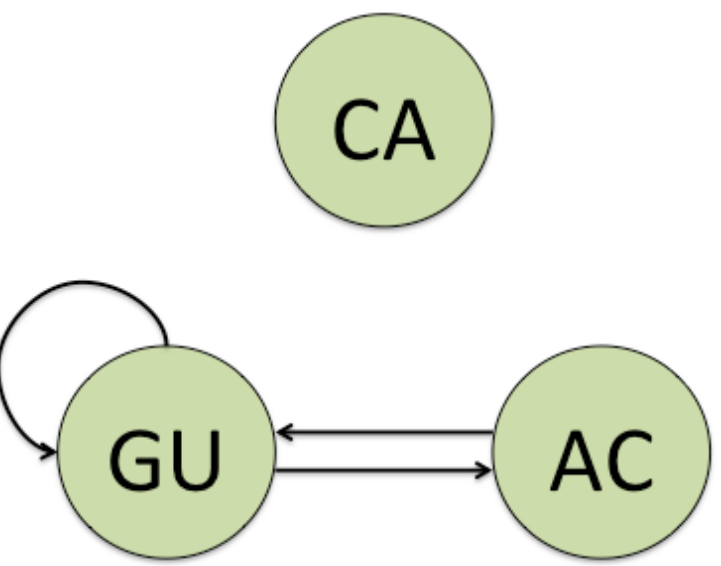

Figure 7: The predicted topological network structure of the genotype triplet $\mathrm{GU} / \mathrm{CA} / \mathrm{AC}$ catalytic system as determined by experimentally determined catalytic rate constants [20] of assembly. This system is topologically organized through an autocatalytic (selfish) node nested within, a cooperative, positive feedback loop. This organizational structure also characterized by the predicted latency of genotype CA.

Figure 7 illustrates the predicted topological architecture of the genotype triplet GU/CA/AC catalytic system. This network is organized through three $M N$ nucleotide, 
base pairing interactions: a $\mathrm{G} \bullet \mathrm{U}$ wobble base pair, mediating the self-assembly of genotype $\mathbf{G U}$, a $\mathrm{G} \bullet \mathrm{C}$ Watson-Crick base pair mediating the cross-assembly of genotype AC by genotype $\mathbf{G U}$, and an $\mathrm{A} \cdot \mathrm{U}$ Watson-Crick base pair, mediating the cross-assembly of genotype GU by genotype AC. The rate constant for the self-assembly of genotype GU is $0.0091 \mathrm{~min}^{-1}$, the rate constant for the cross-assembly of genotype AC is 0.0125 $\min ^{-1}$, and the rate constant for the cross-assembly of genotype $\mathbf{G} \mathbf{U}$ is $0.0319 \mathrm{~min}^{-1}$. The topological organization of the genotype triplet GU/CA/AC system suggests this set will not meet the sufficient criteria to constitute a CAS, as it predicts the latency of genotype CA. Although this set may not be organized in such a way as to reproduce all if its constituent elements, this architectural structure still presents some notable topological features. The graphical representation of this system implies that this set could be reduced to form a smaller, 2-genotype CAS comprising the genotypes GU/AC, organized through a selfish, autocatalytic node, nested within a cooperative, positive feedback loop. Nested topologies, particularly within a strong positive amplification loop, may potentially provide a noteworthy advantage to a CAS. Positive feedback loops are characterized by exponential growth, since the two interdependent nodes (in this case, GU and AC) each have the effect of intensifying the signal of the other. The $\mathbf{G U}$ ribozyme can assemble $\mathbf{A C}$, and in turn the nascent $\mathbf{A C}$ ribozymes immediately begin to assemble more $\mathbf{G} \mathbf{U}$ ribozymes, which subsequently leads to the production of more $\mathbf{A C}$ ribozymes and so on. While exponential amplification may imbue the system with some inherent level of instability resulting from oscillating, chaotic behavior, it does provide a CAS a plausible mechanism to diverge from persisting equilibrium conditions that threaten to halt the dynamic processes that are the proximal drivers emergent phenomena like self- 
organization and chemical evolution. Moreover, the biochemical processes of living systems are characterized by states of disequilibrium under kinetic control. Positive feedback loops may potentially provide sufficiently complex abiotic chemical networks an analogous form of kinetic control. Furthermore, the autocatalytic node nested within the feedback loop could offer a regulatory mechanism to modulate the intensity of the signal being amplified.

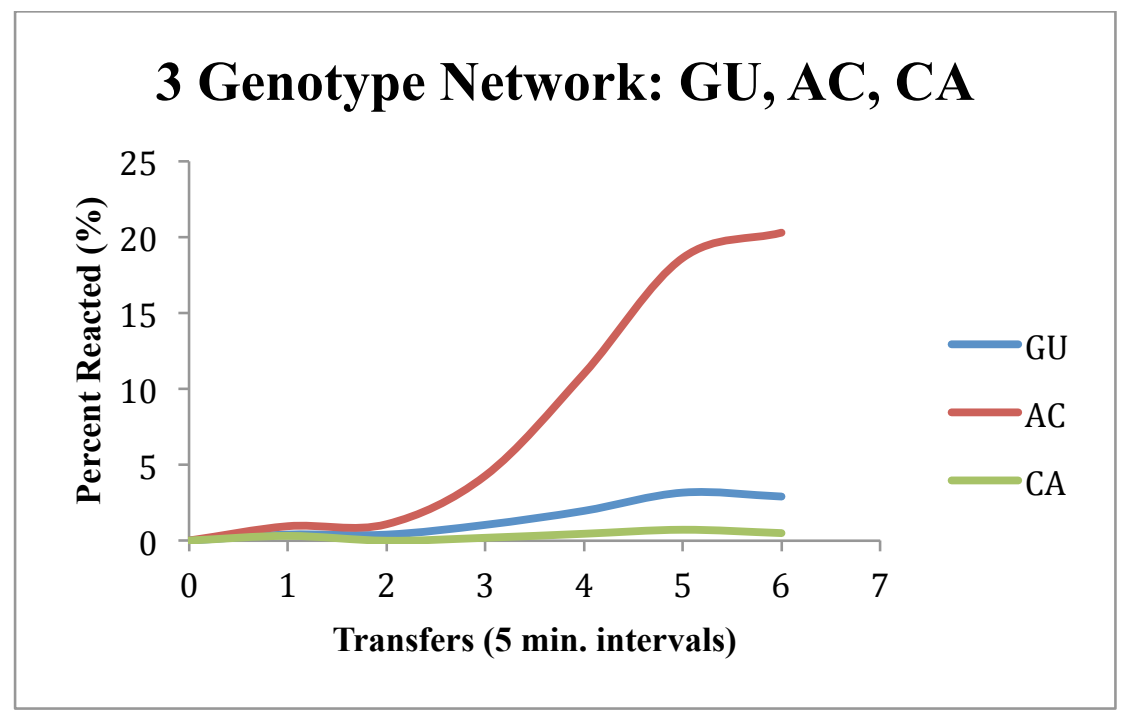

Figure 8: Empirical result from the serial dilution experiment. Catalytic network system composed of the WXY genotypes GU/AC/CA. This plot demonstrates the evolving population dynamics of this triplet set over the course of 6 serial transfers and fluctuations in population diversity are expressed as a percent of original WXY molecules that are assembled into catalytically active $\mathbf{W X Y Z}$ ribozymes.

A plot of the global population outcomes from the serial dilution experiment of the GU/AC/CA triplet set as a function of 6 serial transfers over the duration of 30 minutes (Figure 8). Changes in the global population distribution are represented as the variation in the overall percent of the original WXY fragments that get assembled into full length, WXYZ ribozymes. These data serve to corroborate the topological prediction that the genotype triplet system GU/AC/CA does not form a CAS, as the plot demonstrates there 
is no appreciable assembly of the WXYZ-CA genotype in this reaction network. Unexpectedly however, the positive feedback interplay between the genotypes $\mathbf{G U}$ and AC served to amplify only one of the two nodes within the motif. Moreover, the catalytic pathway leading to the assembly of WXYZ-AC by WXYZ-GU appears to be kinetically preferred, despite a catalytic rate constant that is over $250 \%$ slower than the catalytic assembly rate of WXYZ-GU by WXYZ-AC. This is even without considering kinetic contribution due WXYZ-GU self-assembly, which in theory, should only serve to further diverge the population distributions in favor of genotype GU. It is plausible that some unidentified, non-canonical interaction could be responsible for the peculiarity of these results in this particular catalytic network system although further investigation will be necessary to identify its underlying etiology.

Genotype Triplet Catalytic System: $A C, C G, \& U U$
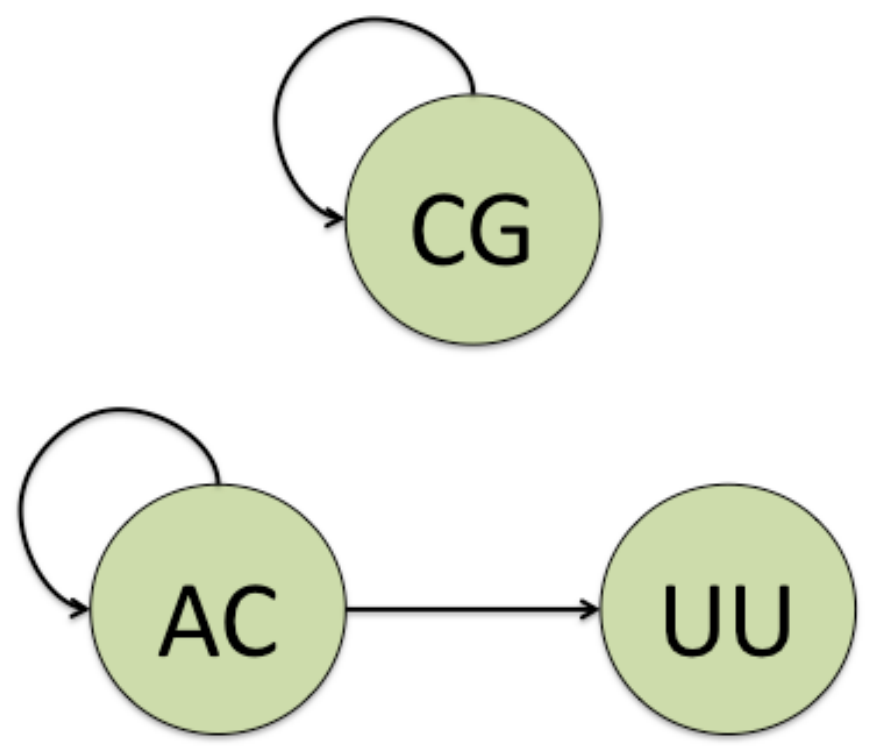

Figure 9: The predicted topological network structure of the genotype triplet $\mathrm{AC} / \mathrm{CG} / \mathrm{UU}$ catalytic system as determined by experimentally determined catalytic rate constants [20] of assembly. This system is topologically organized through two selfish, autocatalytic nodes, with one (AC) being nested within a cooperative, cross-assembly network. 
Figure 9 illustrates the predicted topological architecture of the genotype triplet $\mathrm{AC} / \mathrm{CG} / \mathrm{UU}$ catalytic system. This network is organized through three $M N$ nucleotide, base pairing interactions: an $\mathrm{A} \cdot \mathrm{U}$ Watson-Crick base pair, mediating the cross-assembly of genotype $\mathbf{U U}$ by genotype $\mathbf{A C}$, a $\mathrm{C} \cdot \mathrm{G}$ Watson-Crick base pair mediating the selfassembly of genotype $\mathbf{C G}$, and a non-canonical $\mathrm{A} \cdot \mathrm{C}$ base pair, mediating the selfassembly of genotype AC. The rate constant for the self-assembly of genotype CG is $0.0415 \mathrm{~min}^{-1}$, the rate constant for the cross-assembly of genotype $\mathbf{U} \mathbf{U}$ is $0.0319 \mathrm{~min}^{-1}$, and the rate constant for the self-assembly of genotype $\mathbf{A C}$ is $0.0069 \mathrm{~min}^{-1}$. The organizational architecture of the genotype triplet catalytic system $\mathrm{AC} / \mathrm{CG} / \mathrm{UU}$ suggests a CAS could plausibly emerge from this set, although the relatively slow self-assembly rate of AC may present a significant kinetic obstacle, particularly within the context of a closed system with finite resources. The predicted topology of this network also suggests this may be a reducible system where the CG could be partitioned from the rest of the system, giving rise to two, closed, self-sustaining systems. While the network graph suggests minimal connectivity with a network degree of 4 , the overall lack of interdependence between constituent elements makes this system an unlikely candidate for incorporation into higher levels of organization, although it is certainly not inconceivable. While this triplet system is less than ideal, both because of limited interdependence between the nodes, and due to the relatively low kinetic favorability of AC self-assembly, it does present the opportunity to directly contrast the kinetically favored selfish self-assembly of genotype CG $\left(0.0415 \mathrm{~min}^{-1}\right)$ with the less kinetically facile cooperative cross-assembly of genotype $\mathbf{U U}$ genotype $\mathbf{A C}\left(0.0319 \mathrm{~min}^{-1}\right)$. 


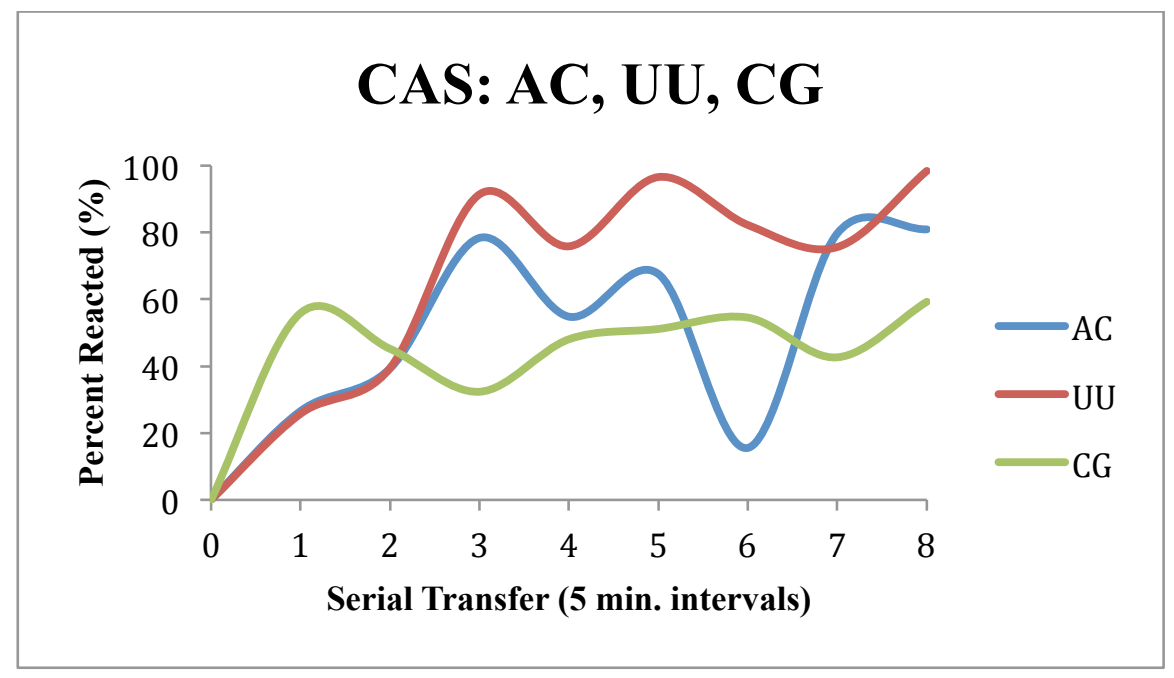

Figure 10: Empirical result from the serial dilution experiment. Catalytic network system composed of the WXY genotypes AC/UU/CG. This plot demonstrates the evolving population dynamics of this triplet set over the course of 8 serial transfers and fluctuations in population diversity are expressed as a percent of original WXY molecules that are assembled into catalytically active $\mathbf{W X Y Z}$ ribozymes.

A plot of the global population outcomes from the serial dilution experiment of the $\mathrm{AC} / \mathrm{UU} / \mathrm{CG}$ triplet system as a function of 8 transfers for a duration of 40 minutes is shown in Figure 10. Changes in the global population distribution are represented as the variation in the overall percent of the original WXY fragments that get assembled into full length, WXYZ ribozymes. This network topology allows for a direct comparison between the selfish assembly pathway of the CG genotype and the cooperative assembly pathway of the genotype UU. From previously determined experimental rate data [20], it appears as though the selfish activity of CG would have over a $130 \%$ kinetic efficiency advantage over the cooperative activity that enables the assembly of $\mathbf{U U}$ by $\mathbf{A C}$. Moreover, it is conceivable that any small kinetic preference toward a single genotype would be amplified in the system over the duration of the experiment through exponential population growth. However, these data suggest otherwise. The UU cooperative 
assembly pathway has a clear kinetic advantage within this system from the second transfer on, and continues for the duration of the experiment (between 10 minutes and 40 minutes of reaction time). Although cooperative assembly in this network is initially sluggish relative to selfish assembly, which peaks to nearly $60 \%$ reacted within the first 5 minutes, its steady growth soon outpaces selfish assembly and continues to climb all the way to its peak at nearly $80 \%$ reacted around 15 minutes into the reaction. Even the slow selfish assembly of AC surpasses CG self-assembly nearly 10 minutes in. A closer examination of the growth curves for the populations of $\mathbf{U} \mathbf{U}$ and $\mathbf{C G}$ reveals a few interesting correlations. Although subtle, it appears as though the curve for $\mathbf{U} \mathbf{U}$ passes through an inflection point between the first and second transfers. It also appears as if this inflection point corresponds with a precipitous decline in the curve for $\mathbf{C G}$ at around the same time. Continuing along both curves reveals that $\mathbf{U} \mathbf{U}$ reaches a local maximum around the third transfer while simultaneously CG is approaching its local minimum. While further experimentation is required to attribute causality to these observations, it is plausible the cooperative interplay between $\mathbf{A C}$ and $\mathbf{U U}$ potentially has an inhibitory effect on the growth of the CG in this system, although a mechanism by which this could occur is currently unclear. It does seem however, participation in cooperative pathways does proffer, an as of yet unquantified, kinetic advantage that is greater than the additive sum of the rate constants of the members of the cooperative network. Finally, it should be noted that the sudden drop observed by the AC reaction curve at transfer 6 is likely due to a quantification error as it seems unlikely that the slow reproduction of $\mathbf{A C}$ could result in the rapid growth observed between transfers 6 and 7 . 

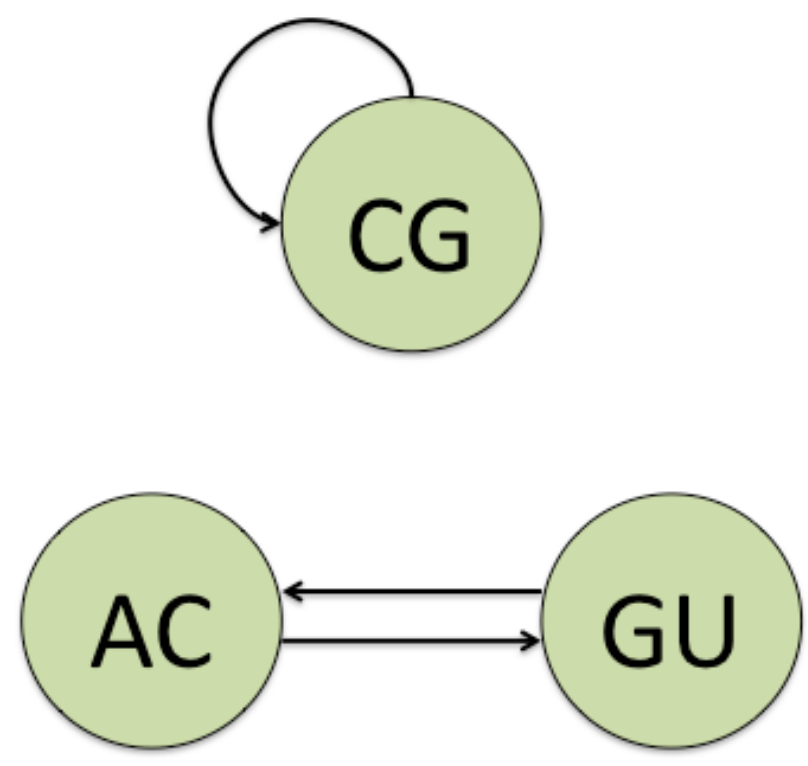

Figure 11: The predicted topological network structure of the genotype triplet $\mathrm{AC} / \mathrm{CG} / \mathrm{GU}$ catalytic system as determined by experimentally determined catalytic rate constants [20] of assembly. This system is topologically organized through a selfish, autocatalytic node and a mutually cooperative feedback loop.

Figure 11 illustrates the predicted topological architecture of the genotype triplet $\mathrm{AC} / \mathrm{CG} / \mathrm{GU}$ catalytic system. This network is organized through three $M N$ nucleotide, base pairing interactions: a $\mathrm{C} \cdot \mathrm{G}$ Watson-Crick base pair, mediating the self-assembly of genotype $\mathbf{C G}$, an $\mathrm{A} \cdot \mathrm{U}$ Watson-Crick base pair mediating the cross-assembly of genotype GU by genotype $\mathbf{A C}$, and a $\mathrm{G}^{\bullet} \mathrm{C}$ Watson-Crick base pair, mediating the cross-assembly of genotype AC by genotype $\mathbf{G U}$. The rate constant for the self-assembly of genotype CG is $0.0415 \mathrm{~min}^{-1}$, the rate constant for the cross-assembly of genotype $\mathbf{G U}$ is 0.0319 $\min ^{-1}$, and the rate constant for the self-assembly of genotype $\mathbf{A C}$ is $0.0125 \mathrm{~min}^{-1}$. The network topology of this three-genotype set is the composition of a selfish, autocatalytic node, with a mutually cooperative feedback loop. Once again, based on experimental rate data [20], the selfish $\mathbf{C G}$ node has a distinct kinetic advantage over both cross-assembly 
reactions within the feedback loop, however it seems reasonable to expect the kinetics of the feedback mechanism to enhance the rate at which both $\mathbf{A C}$ and $\mathbf{G U}$ are assembled, however it is unclear as to how much. This networks structure again allows for a direct comparison of a mutually cooperative network motif against a selfish node to further corroborate the original premise, that cooperative interactions are given kinetic preferentiality in closed systems, over selfish interactions. To a first approximation, the interactions in this set are mediated exclusively through Watson-Crick base pairing interactions as discussed above. This network also displays only modest connectivity with a cumulative degree of 5 , four of which are attributed to the feedback interaction. This set is also predicted to form a CAS, however the structure suggests this system would be reducible by partitioning the CG node, putatively resulting in two selfsustaining systems.

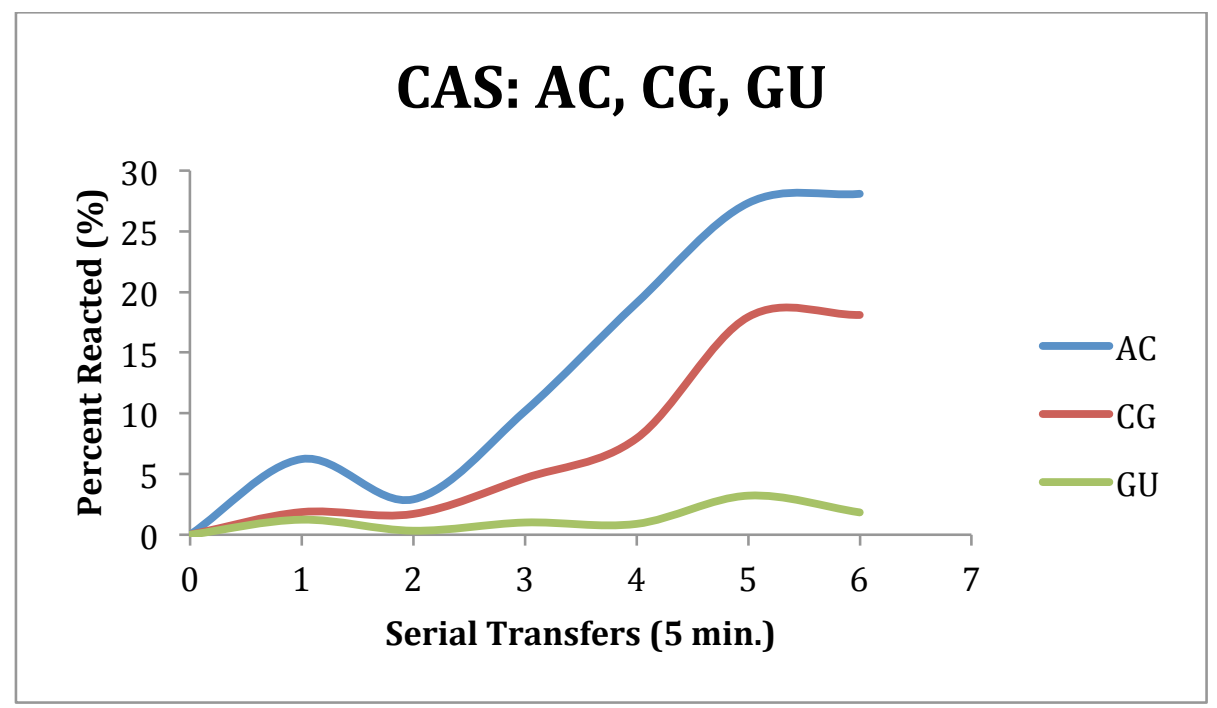

Figure 12: Empirical result from the serial dilution experiment. Catalytic network system composed of the WXY genotypes AC/CG/GU. This plot demonstrates the evolving population dynamics of this triplet set over the course of 6 serial transfers and fluctuations in population diversity are expressed as a percent of original WXY molecules that are assembled into catalytically active $\mathbf{W X Y Z}$ ribozymes. 
A plot of the global population outcomes from the serial dilution experiment of the $\mathrm{AC} / \mathrm{UU} / \mathrm{CG}$ triplet system as a function of 8 transfers for a duration of 40 minutes is shown in Figure 12. Changes in the global population distribution are represented as the variation in the overall percent of the original WXY fragments that get assembled into full length, WXYZ ribozymes. Similar to a previously described system, this triplet set allows for the direct contrast of a selfish node against a mutually cooperative, two-node feedback loop. This system slightly differs from the previous example however, in that this set is simply a composition of the two motifs with no observable nested structures. The population growth curves for this system provide another data point in direct comparison of selfish and cooperative network modules, and behave in accordance to previous observations that cooperative network modules offer a kinetic advantage to those genotypes that participate in them. This is again inferred because the outcomes of these serial dilution network experiments are in contrast to the kinetic efficiency these ribozymes display during kinetic assays, in isolation. CG self-assembly has been determined to one of the more facile assembly reaction among the 16 mutant Azoarcus mutants, however in a network context with two genotypes that also readily engage in mutual cross-assembly reactions through strong Watson-Crick interactions, CG selfassembly simply does not proceed with the same expedience as it does in isolated kinetic studies [20]. Moreover, $\mathbf{A C}$ cross-assembly by $\mathbf{G U}$ proceeds far more efficiently than expected from the same isolated kinetic studies [20]. Again, it is interesting that the positive feedback loop does not amplify both genotypes involved, and in this case kinetic preferentiality is given to the assembly process with the slowest experimental rate constant. In this, and the previously described system, we speculate that non-canonical 
interactions conceivable within the system may play a role in the kinetic rate enhancement observed relative to other genotypes within the system. A plausible $A \cdot C$ base pair that can form between the Watson-Crick base pairing surfaces of an adenosine residue and a cytidine residue may discreetly influence the dynamics of these small systems, although further investigation is necessary before more conclusive inferences can be drawn.

\section{Conclusion}

It has long been speculated that a fully functional RNA world mediated a transition to the DNA/protein based biochemical processes observed in contemporary biological systems, by providing both a pre-existing infrastructure and by exercising a form of kinetic control such that a transition DNA/proteins could be selected. It is still unclear however, what plausible chemical mechanisms allow for the decentralized types of hierarchical organization necessary to facilitate this event(s). Emerging evidence, in accordance with existing postulation, suggests that collectively autocatalytic systems (CAS) may have been able to provide the architecture necessary through spontaneously self-organizing networks. Moreover, if self-organizing structures do arise through sufficiently complex chemical systems, they would likely be under kinetic control as systems tending toward thermodynamic equilibrium exhibit increasing levels of disorganization as a consequence of entropic gain. In this manuscript, we describe how graph theoretic models derived from experimental kinetic data can inform the topological structure of collectively autocatalytic systems. Furthermore, we characterize four, 3-genotype networks, arising from spontaneously self-assembling oligonucleotide fragments of the Azoarcus ribozyme 
recombinase system. Finally, we illustrate a few contexts where network topology can either enhance or diminish the kinetic efficiency of the recombination reactions catalyzed by the Azoarcus ribozyme, providing an example of the plausible causality between topological organization and kinetic control. 


\section{$\underline{\text { References }}$}

1. Robertson, M.P., Joyce, G.F., Highly efficient self-replicating RNA enzymes., Chemical Biology., (2014) 21(2): 238-245.

2. Hayden, E.J., Lehman, N., Self-assembly of a group I intron from inactive oligonucleotide fragments., (2006) 13(8): 909-918.

3. Jayathilaka, T.S., Lehman, N., Spontaneous covalent self-assembly of the Azoarcus ribozyme from five fragments., (2018) 19(3): 217-220.

4. Plaxco, P.W., Gross, M., Astrobiology: A Brief Introduction Second Edition., Johns Hopkins University Press., (2011) 78-92.

5. Glasco, D.M., Beyond the DNA-protein paradox: a "clutch" of other chicken-egg paradoxes in cell and molecular biology., Answers Research Journal., (2016) 9: 209-227.

6. Nissen, P., Hansen, J., Ban, N., Moore, P.B., Steitz, T.A., The structural basis of ribosome activity in peptide bond synthesis. Science Magazine.,(2000) 289: 920929.

7. Lerner, M.R., Boyle, J.A., Mount, S.M., Wolin, S.L., Steitz, J.A., Are snRNPs involved in splicing?, Nature., (1980) 283, 220-224.

8. Nelson, David L., Cox, Michael M., Lehninger Principles of Biochemistry., $6^{\text {th }}$ ed. New York: W.H. Freeman, 2013. Print.

9. Orgel, L.E., Prebiotic Chemistry and the origin of the RNA world., Critical Reviews in Biochemistry and Molecular Biology., (2004) 39: 99-123.

10. Kruger, K., Grabowski, P.J, Zaug, A.J., Sands, J., Gottschling, D.E., Cech, T.R., Self-splicing RNA: autoexcision and autocyclization of the ribosomal RNA intervening sequence of Tetrahymena., Cell., (1982) 31(1): 147-157.

11. Guerrier-Takada, C., Gardiner, K., Marsh, T., Pace, N., Altman, S., The RNA moiety of Ribonuclease $\mathrm{P}$ is the catalytic subunit of the enzyme., Cell., (1983) 35(3 Pt 2): 849-857.

12. Alberts, B., Johnson, A., Lewis, J., Molecular Biology of the Cell: The RNA World and the Origins of Life., $4^{\text {th }}$ ed. New York: Garland Science., (2002). Print.

13. Hordijk, W., Autocatalytic sets: from the origin of life to the economy., Bioscience., (2013) 63(11): 877-881.

14. Hordijk, W., Vaidya, N., Lehman, N., Serial transfer can aid the evolution of autocatalytic sets., Journal of Systems Chemistry., (2014) 5:4.

15. Eigen, M., Schuster, P., The hypercycle., Naturwissenschaften., (1978) 65(1): 741.

16. Hordijk, W., Kauffman, S., Seel, M., Required levels of catalysis for the emergence of autocatalytic sets in models of chemical reaction systems., International Journal of Molecular Sciences., (2011) 12(5): 3085-3101.

17. Vaidya, N., Manapat, M., Chen, I.A., Xulvi-Brunet, R., Hayden, E.J., Lehman, N., Spontaneous network formation among cooperative RNA replicators., Nature., (2012) 491(7422).

18. Pross, Addy., What is Life? How Chemistry Becomes Biology., Oxford University Press, Oxford, UK., (2012) 58-81. Print. 
19. Hordijk, W., Steel, M., Dittrich, P., Autocatalytic sets and chemical organizations: modeling self-sustaining reaction networks at the origin of life. New Journal of Physics., (2018) 20(015011).

20. Yeates, J.A., Hilbe, C., Zwick, M., Nowak, M.A., Lehman, N., Dynamics of prebiotic RNA reproduction illuminated by chemical game theory., Proceedings of the National Academy of Sciences USA., (2016) 113(18): 5030-5035.

21. Mathis, C., Ramprasad, S.N., Walker, S.I., Lehman, N., Prebiotic RNA network formation: taxonomy of molecular cooperation., Life., (2017) 7(4): 2-11.

22. Nghe, P., Hordijk, W., Kauffman, S.A., Walker, S.I., Schmidt, F.J., Kemble, H., Yeates, J.A., Lehman, N., Prebiotic network evolution: Six key parameters., Mol. BioSyst., (2015) 11: 3206-3217.

23. Reinhold-Hurek, B., Shub, D.A., Self-splicing introns in tRNA genes of widely divergent bacteria., Nature., (1992) 357: 173-176.

24. Ingber, D.E., The origin of cellular life., BioEssays., (2000) 22: 1160-1170.

25. Wirth, N., Algorithms + data structures = programs., Prentice Hall PTR, Upper Saddle River, NJ, USA., (1978) pp. 126.

26. Kaisler, S.H., Madey, G., Complex and adaptive systems: emergence and selforganization., Hawaii International Conference on System Sciences \#42, Big Island, HI, (2009).

27. Gignoux, J., Cherel, G., Davies, I.D., Flint, S.R., Lateltin, E., Emergence and complex systems: the contribution of dynamic graph theory., Ecological Complexity., (2017) 31: 34-49.

28. Zamani, M., Vicsek, T., Glassy nature of hierarchical organizations. Scientific Reports., (2017) 7(1382): 1-9.

29. Ravasz, E., Barabasi, A., Hierarchical organization in complex networks., Physical Review E., (2003) 67(2): 026112.

30. Hlinka, J., Hartman, D, Palus, M., Small-world topology of functional connectivity in randomly connected dynamical systems., Chaos: An Interdisciplinary Journal of Nonlinear Science., (2012) 22: 033107.

31. Mason, O., Verwoerd, M., Graph theory and networks in biology., IET Systems Biology., (2007) 1(2): 89-119. 